\title{
Modeling particle transport and discoloration risk in drinking water distribution networks
}

\author{
Joost van Summeren and Mirjam Blokker \\ KWR Watercycle Research Institute, Nieuwegein, 3433PE, the Netherlands \\ Correspondence to: Joost van Summeren (joost.van.summeren@kwrwater.nl) \\ Received: 15 March 2017 - Discussion started: 2 May 2017 \\ Revised: 29 July 2017 - Accepted: 6 October 2017 - Published: 16 October 2017
}

\begin{abstract}
Discoloration of drinking water is a worldwide phenomenon caused by accumulation and subsequent remobilization of particulate matter in drinking water distribution systems (DWDSs). It contributes a substantial fraction of customer complaints to water utilities. Accurate discoloration risk predictions could improve system operation by allowing for more effective programs on cleaning and prevention actions and field measurements, but are challenged by incomplete understanding on the origins and properties of particles and a complex and not fully understood interplay of processes in distribution networks. In this paper, we assess and describe relevant hydraulic processes that govern particle transport in turbulent pipe flow, including gravitational settling, bedload transport, and particle entrainment into suspension. We assess which transport mechanisms are dominant for a range of bulk flow velocities, particle diameters, and particle mass densities, which includes common conditions for DWDSs in the Netherlands, the UK, and Australia. Our analysis shows that the theoretically predicted particle settling velocity and threshold shear stresses for incipient particle motion are in the same range as, but more variable than, previous estimates from lab experiments, field measurements, and modeling. The presented material will be used in the future development of a numerical modeling tool to determine and predict the spatial distribution of particulate material and discoloration risk in DWDSs. Our approach is aimed at understanding specific causalities and processes, which can complement data-driven approaches.
\end{abstract}

\section{Introduction}

Discoloration events in drinking water distribution networks occur worldwide as a result of accumulation of particulate material in pipes and subsequent remobilization due to hydraulic disturbances (e.g., Vreeburg, 2007). Strategies to reduce the risk of discoloration events include quality improvement of treated water (to reduce the particle load into the network), implementation of a self-cleaning network layout (to prevent particle accumulation), and cleaning actions (to locally remove accumulated material from pipes before the amount becomes too high). Although these strategies are partly successful, the processes and mechanisms that cause discoloration events are complex and not yet fully understood, which hampers further risk reduction. For example, advanced treatment processes have enhanced the water quality that enters distribution systems (DWDSs), but cannot fully remove the load of particulate matter that enters
DWDSs. The resuspension potential method has improved the understanding in the local presence of particulate matter and the relationship with hydraulic events, but does not provide a comprehensive understanding of particulate matter transport (Vreeburg et al., 2005). Self-cleaning networks have been implemented and found to be effective in reducing discoloration risks in the Netherlands (Blokker et al., 2009), but have only been implemented in some Dutch DWDSs installed after the mid-1990s.

Field measurements from an extensive and prolonged program of cleaning actions that covers $\sim 450 \mathrm{~km}$ of pipes per year by Dutch water company PWN indicate that highturbidity events often occur in repeatable spatial and temporal patterns (Blokker and Schaap, 2011; Mounce et al., 2016). Factors that influence these patterns include the hydraulic vigor associated with the buildup and displacement of particles (Blokker and Schaap, 2007) and sediment load 
from the treatment plant and transport mains upstream from the DWDS (Blokker and Schaap, 2015a). Although not all of the observed variability in particle accumulation can fully be explained, the repeatability suggests the possibility of describing the propagation and spatial distribution of particle cumulates in the DWDS with a model.

Several models for discoloration in DWDSs exist. Richardt et al. (2009) developed a model to calculate flushing intervals aimed at preventing the deposit growth in pipe segments (inferred from measured deposits) from exceeding the maximum possible deposit level (derived from the maximum daily velocity from a hydraulic simulation). A decrease in customer complaints was reported when applied to a reallife network. One drawback of their approach is that it requires deposition information for all pipe segments in the study area. Another is that transfer of deposits across network segments is not allowed for. As such, changing flow conditions - e.g., flow reversals due to anomalous supply conditions - will pose a challenge to the quality of the model results.

The Particle Sediment Model (PSM, Ryan et al., 2008) includes the processes of particle settling, resuspension, and wall deposition across the complete pipe circumference. The Variable Condition Discoloration Model (VCDM, Furnass et al., 2014) builds on the Prediction and Control of Discolouration in Distribution Systems (PODDS, Boxall et al., 2001). VCDM assumes that discoloration is related to the attachment and erosion of particles to pipe walls. It provides a mathematical formulation for the simultaneous occurrence of erosion of weak material (i.e., material with a strength below the actual shear stress) and build-up of strong material (strength above the actual shear stress). VCDM has been validated for individual transport mains, but not for smaller distribution pipes, for which it is plausible that gravitational settling dominates. Although PSM and VCDM are partly successful in predicting patterns of local particle accumulation (e.g., Vogelaar and Blokker, 2010; Furnass et al., 2014), this success depends strongly on model calibration with local field measurements and laboratory data (for the Australian and British utilities, respectively). Available transport may be incomplete because of the absence of potentially important processes, such as bed-load transport. In addition, conditions and mechanisms may differ from DWDSs for which the model was not calibrated, such as different source quality due to other treatment processes or non-chlorinated Dutch systems that include self-cleaning network layouts.

The objective of the present paper is to develop a theoretical framework that describes the leading processes governing particle transport and causing discoloration events. The framework serves as a basis for developing a numerical modeling tool to determine and predict the spatial distribution of particulate material in (Dutch) drinking water distribution systems. System operation can benefit from successful predictions that allow for more effective programs on cleaning actions and field measurements, particularly in network areas where available data are limited or absent. Because field data indicate that multiple transport mechanisms (e.g., gravity and turbulence) and modes (e.g., bed-load transport and transport in suspension) can operate simultaneously or in succession, a model based on a description of processes rather than extrapolation of available measurements is more likely to result in reliable and generically applicable predictions. We assess which particle transport mechanisms are dominant for a range of drinking water conditions and particle properties, in particular flow velocity, particle diameter, and particle size. This serves as a basis for later development of a numerical tool for the transport of particulate material. Our approach is aimed at understanding specific causalities and processes. This can complement data-driven approaches which give important understanding in key descriptive parameters for particular DWDSs (e.g., Mounce et al., 2016) but do not investigate the causalities and processes explicitly.

\section{Identifying relevant transport processes from current understanding of particle transport in DWDSs}

Several previous studies have demonstrated the importance of processes and mechanisms within DWDSs, in addition to processes at treatment plants and transport mains, to determine particle transport and discoloration risk. Laboratory experiments with smooth pipes have demonstrated that at low flow velocity only the lower half of pipes accumulate particles (iron flocs), while at a higher flow velocity, particles accumulate over the complete pipe circumference (Vreeburg, 2007; Ryan et al., 2008). Circumferential accumulation is likely associated with the process of turbophoresis; its contribution is mostly limited to large particles at high flow velocities in transport mains and is not expected to play a major role in distribution mains (Van Thienen et al., 2011). This study shows that turbophoresis is predicted to significantly contribute to radial sediment transport for flow velocities of $>1 \mathrm{~m} \mathrm{~s}^{-1}$ for $\sim 50 \mu \mathrm{m}$ particles and even higher flow velocities for smaller particles. Accumulation of particles on the pipe's lower half can plausibly be attributed to settling under the influence of gravitational acceleration.

DWDS pipelines with maximum daily flow velocities above 0.2 to $0.25 \mathrm{~m} \mathrm{~s}^{-1}$ are less prone to particle accumulation, plausibly because daily erosion of sediments prevents build-up to significant discoloration risk levels, and this has led to the successful implementation of self-cleaning networks with pipeline diameters adjusted to attain this flow velocity in the Dutch drinking water sector (Vreeburg, 2007). The importance of hydraulic processes within the DWDS is further corroborated by evidence that the self-cleaning capacity can be regulated by valve manipulation, and measurements suggest an influence of the vertical inclinations of pipes, pipe junctions, and plumbing fixtures (related to valves, hydrants, sensors, etc.; Schaap and Blokker, 2013). In 


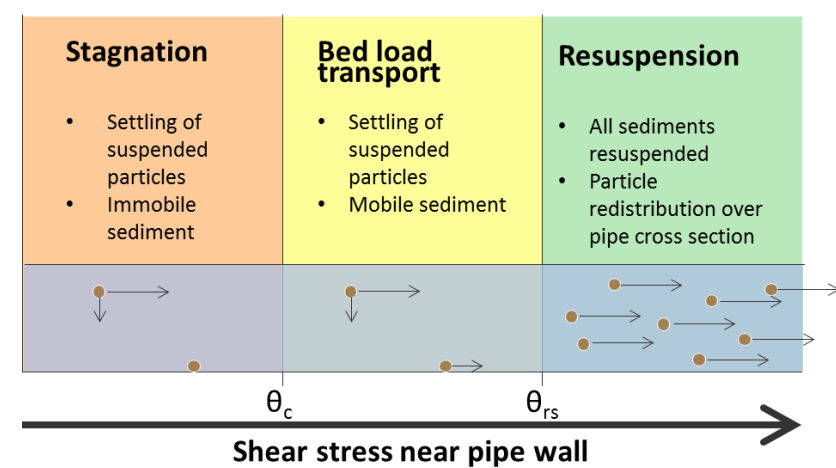

Figure 1. Conceptual representation of the particle transport model. Below a dimensionless critical shear stress (Shields number for incipient motion, $\theta_{\mathrm{c}}$, red area) particles in suspension settle to the bottom of the pipe under the influence of gravity and, once settled, remain immobile. Above the critical Shields number for particle entrainment $\left(\theta_{\mathrm{rs}}\right.$, green area), we assume that all particles are resuspended instantly and redistributed uniformly over the cross section of the pipe. At intermediate shear stress conditions (yellow area), suspended particles settle and particles in the sediment phase move by means of bed-load transport. The transport phases are schematically visualized in the blue ribbon.

addition, independent studies suggest that sediment load and frequency of customer discoloration reports vary with the water temperature within the distribution pipes, as opposed to temperatures at the treatment plant (Blokker and Schaap, 2015b; Van Summeren et al., 2015). Furthermore, a cluster analysis of discoloration customer reports shows evidence that discoloration events are caused on the local level, suggesting that mechanisms in distribution pipelines rather than in transport mains critically control discoloration events (Van Rooij, 2016). Simultaneous lance measurements at various pipe depths suggest a downward positive concentration gradients during elevated turbidity events, suggesting the possibility of sediment mobilization through bed-load transport in distribution pipes (Schaap and Kivit, 2007).

With the above in mind, we decided to focus on a model for distribution pipelines that captures particle settling under the influence of gravity, particle resuspension by hydraulic forces, and sediment mobility by means of bed-load transport. We investigate how particle size, particle density and flow velocity, and associated shear stresses affect these processes.

Other mechanisms related to turbulent motion-particle interaction are likely relevant but probably not dominant. Turbulent diffusion can perturb the trajectory of a suspended particle and, hence, its apparent settling velocity. Turbulent dispersion effects near the viscous sub-layer can also modulate particle concentration profiles. Turbulent fluctuations can influence incipient motions and resuspension, but are only implicitly incorporated into our analysis.

Particulate accumulation is known to be related to biological activity (Gauthier et al., 1999). Organic matter in particulate accumulation may consist of $1-12 \%$ of bacterial biomass, making the deposits an important factor in biological safety of drinking water (Vreeburg, 2007). However, because interactions between particle transport and microbiological processes are complex and not yet fully understood, they are kept out of the scope of the current research. Cementation or armoring and compaction of sediment layers potentially strengthen sediments over time, but this aspect is out of the scope of this paper. Chemical processes such as flocculation may change the particles' size and density during transport. Consideration of this process requires detailed knowledge of particle properties and the local water chemistry and might be added at a later stage.

\section{Model description for particle transport}

\subsection{General remarks}

The particle transport model is outlined in the sections below and conceptually visualized in Fig. 1. Parameter domains for stagnation, bed-load transport, and resuspension are separated by threshold values of a dimensionless shear stress (Shields number, $\theta$ ). Key parameters used in this study are listed in Table 1.

\subsection{Gravitational settling of particulate material}

We assume that at subcritical shear stress conditions (further described in Sect. 3.2) particles in the water phase sink under the influence of gravity, with a settling velocity $u_{\mathrm{s}}$ and particles that reach the pipe wall remaining immobile. The settling (or fall) velocity is formulated by Stokes' law, first stated in 1851 , which describes the balance between gravity, buoyancy, and friction of a sinking particle. The settling velocity depends on particle properties and hydraulic conditions and is assumed to be independent of the larger-scale flow. The general form of the free (no interaction between individual particles) settling velocity for spherical, incompressible particles is

$u_{\mathrm{s}}=\left[\frac{4 g s d_{\mathrm{p}}}{3 C_{\mathrm{d}}}\right]^{1 / 2}$,

with $C_{\mathrm{d}}$ the friction coefficient, $g$ the gravitational acceleration, $s \equiv\left(\rho_{\mathrm{p}}-\rho_{\mathrm{f}}\right) / \rho_{\mathrm{f}}$ the relative excess mass density, $\rho_{\mathrm{p}}$ and $\rho_{\mathrm{f}}$ the particle and fluid density, respectively, and $d_{\mathrm{p}}$ the particle diameter. In case of a small particle Reynolds number $(<0.2)$, the fluid flow surrounding the particle is laminar, independent of the larger-scale fluid flow:

$R e_{\mathrm{p}}=\frac{u_{\mathrm{s}} d_{\mathrm{p}}}{v(T)}<0.2$, 
Table 1. Definitions of key parameters. Symbol $d_{\mathrm{p}}$ is used for the particle diameter, $\rho_{\mathrm{p}}$ and $\rho_{\mathrm{f}}$ represent mass particle and fluid density, respectively, $\kappa$ is the von Kármán constant, $v$ is the (temperature-dependent) kinematic viscosity of water, $g$ is the gravitational acceleration (taken as $9.81 \mathrm{~ms}^{2}$ ), $C_{\mathrm{d}}$ is the friction factor, and $C_{\mathrm{f}}$ is the Darcy friction factor (taken as 0.02 in this study unless otherwise mentioned).

\begin{tabular}{lll}
\hline Parameter description & Formulation & Unit \\
\hline Particle Reynolds number & $R e_{\mathrm{p}}=\frac{u_{\mathrm{s}} d_{\mathrm{p}}}{v}$ & - \\
Boundary Reynolds number & $R e_{*}=\frac{u_{*} d_{\mathrm{p}}}{v}$ & - \\
Rouse number & $P=\frac{u_{\mathrm{s}}}{\kappa u_{*}}$ & - \\
Relative specific particle weight & $s=\frac{\rho_{\mathrm{p}}-\rho_{\mathrm{f}}}{\rho_{\mathrm{f}}}$ & - \\
Particle settling velocity (general form) & $u_{\mathrm{s}}=\left[\frac{4 g s d_{\mathrm{p}}}{3 C_{\mathrm{d}}}\right]^{1 / 2}$ & $\mathrm{~m} \mathrm{~s}^{-1}$ \\
Particle settling velocity (for particle Reynolds numbers) & $u_{\mathrm{s}}=\frac{g s d_{\mathrm{p}}^{2}}{18 v_{\tau_{\mathrm{b}}}}$ & $\mathrm{m} \mathrm{s}^{-1}$ \\
Shields number & $\theta=\frac{\left.\rho_{\mathrm{p}}-\rho_{\mathrm{f}}\right) g d_{\mathrm{p}}}{}$ & - \\
Critical Shields number for particle incipient motion & $\theta_{\mathrm{c}}$ & - \\
Critical Shields number for particle entrainment & $\theta_{\mathrm{rs}}$ & - \\
Shear velocity & $u_{*}=u_{f} \sqrt{C_{\mathrm{f}}}$ & $\mathrm{m} \mathrm{s}^{-1}$ \\
Shear stress near sediment bed & $\tau_{\mathrm{b}}=\frac{\rho_{\mathrm{f}} u_{f}^{2} C_{\mathrm{f}}}{8}$ & $\mathrm{~Pa}$ \\
\hline
\end{tabular}

Table 2. Overview of common particle diameter and density and bulk flow velocity conditions in DWDS for the Netherlands, the UK, and Australia.

\begin{tabular}{|c|c|c|c|}
\hline DWDS & $\begin{array}{r}\text { Particle diameter } \\
(\mu \mathrm{m})\end{array}$ & $\begin{array}{r}\text { Particle density } \\
\left(\mathrm{kg} \mathrm{m}^{-3}\right)\end{array}$ & $\begin{array}{l}\text { Bulk flow velocity } \\
\text { of water }\left(\mathrm{m} \mathrm{s}^{-1}\right)\end{array}$ \\
\hline Dutch & $3-12^{*}$ & $1000-1300$ & $0.05-0.2^{*}$ \\
\hline UK & $5-30 *$ & $1000-1300^{*}$ & $0.05-0.2^{*}$ \\
\hline Australian & $0.5-500$; median: $8-27$ & $1180-2040$ & $0.05-0.2$ \\
\hline
\end{tabular}

with $v(T)$ the temperature-dependent kinematic viscosity. Under these conditions the friction coefficient is given by

$C_{D}=\frac{24 \alpha_{\mathrm{sh}}}{R e_{\mathrm{p}}}$

with $\alpha_{\text {sh }}$ the particle shape factor. We assumed spherically shaped particles, i.e., $\alpha_{\mathrm{sh}}=1$, because particle shape is difficult to determine. Substitution of the friction coefficient in Eq. (1) results in

$u_{\mathrm{s}}=\frac{g s d_{\mathrm{p}}^{2}}{18 v(T)}$

The temperature dependence of the viscosity is relevant, because a temperature increase from 5 to $25^{\circ} \mathrm{C}$ can lower the fluid viscosity and increase the settling velocity by $\sim 70 \%$, as pointed out by Kris and Hadi (2007). We use the free-fall velocity, because hindered settling, whereby the presence of surrounding particles decreases the settling velocity, can be ignored at typical particle concentrations in the ppm range.
Next, we assess expected settling velocities for DWDS conditions documented for the Netherlands, the UK, and Australia (Table 2). Note that the particle diameter range for Australian utilities is comparatively wide, because we used the full range between minimum and maximum diameters documented for 20 locations, and the estimates include transport mains, as opposed to the estimates for the Netherlands and the UK. We represented settling velocities in a contour plot (Fig. 2), which shows a strong positive dependence of the settling velocity on particle size and density: even within a limited density range of $1100-1300 \mathrm{~kg} \mathrm{~m}^{-3}$, the settling velocity for common Dutch DWDS conditions varies over 1 order of magnitude. A default settling velocity of $u_{\mathrm{s}}=1.6 \times 10^{-6} \mathrm{~m} \mathrm{~s}^{-1}$ was determined from experiments of the Australian DWDS (Ryan et al., 2008), indicated by the blue line. Although the default value falls within the conditions of the three countries, a large variation of settling velocities may be anticipated, because of the strong sensitivity to particle properties.

It was pointed out by Ryan et al. (2008) that the empirically derived settling velocity is higher than the Stokes velocity. Possibly, this is because they used a specific gravity derived from dried samples, whereas the (effective) density of suspended particles will be reduced due to the virtual mass effect, whereby a fluid boundary layer encloses a particle and acts as part of the particle in terms of inertia. 


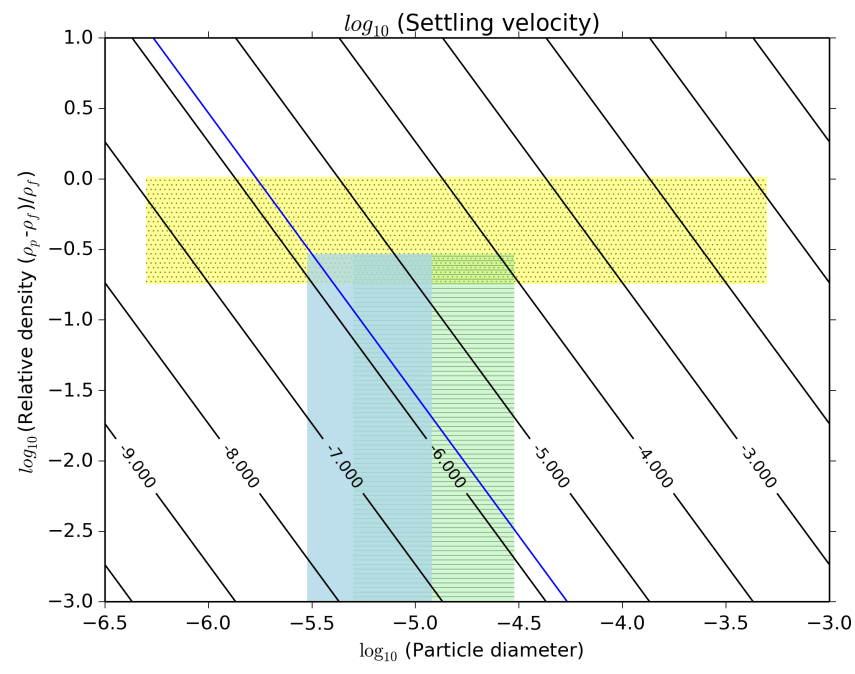

Figure 2. Contour plot of the settling velocity of particles $\left(u_{\mathrm{S}}\right.$ in $\left.\mathrm{m} \mathrm{s}^{-1}\right)$ as a function of particle diameter $\left(d_{\mathrm{p}}\right.$ in $\left.\mathrm{m}\right)$ and particle excess density relative to water $\left(s=\left(\rho_{\mathrm{p}}-\rho_{\mathrm{f}}\right) / \rho_{\mathrm{f}}\right.$, dimensionless). The blue, green-striped, and yellow-dotted rectangles refer to particle properties that are common for Dutch, UK, and Australian DWDSs (see Table 2 and the text for explanation). The black contour lines refer to particle settling velocity values. The blue line refers to the default particle settling velocity of $u_{\mathrm{s}}=1.6 \times 10^{-6} \mathrm{~m} \mathrm{~s}^{-1}$ determined from experiments of the Australian DWDS.

\subsection{Critical wall shear stress for incipient motion and resuspension of particles}

Initiation of particle motion is often determined using a nondimensionalization of the shear stress, known as the Shields number $\theta$ (Shields, 1936):

$\theta=\frac{\tau_{*}}{\left(\rho_{\mathrm{p}}-\rho_{\mathrm{f}}\right) g d_{\mathrm{p}}}=\frac{u_{f}^{2} C_{\mathrm{f}}}{8 s g d_{\mathrm{p}}}$.

The Shields number gives the ratio of shear force exerted on the particle by the fluid and the particle's mass density. In principle, particle motion initiates when the shear stress near the sediment bed becomes larger than a critical value, $\theta>\theta_{\mathrm{c}}$. In the sediment transport literature the Shields diagram gives an empirical relationship for $\theta_{\mathrm{c}}$ as a function of the boundary Reynolds number $R e_{*}=\frac{u_{*} d_{\mathrm{p}}}{v}$ (Shields, 1936; Arolla and Desjardins, 2015). Although the Shields diagram is based on experiments with rectangular channels, it provides a starting point for cylindrically shaped pipes.

Figure 3 shows how larger fluid velocity and smaller particles promote the propensity to incipient particle motion (increasing $\theta)$. For increasing particle size, $d_{\mathrm{p}}\left(1 \times 10^{-6}\right.$ to $1 \times 10^{-5} \mathrm{~m}$ ), and fluid flow velocity, $\bar{u}_{f}\left(0.01\right.$ to $0.1 \mathrm{~m} \mathrm{~s}^{-1}$ ), the boundary Reynolds number increases from $1.4 \times 10^{-3}$ to 14 . The latter value is associated with a $\tau_{\mathrm{c}}$ value of $\approx 0.05$. The former value lies outside the Shields diagram. As a starting point, we assume a value of $\tau_{\mathrm{c}}=10$. The $\log _{10}$-values

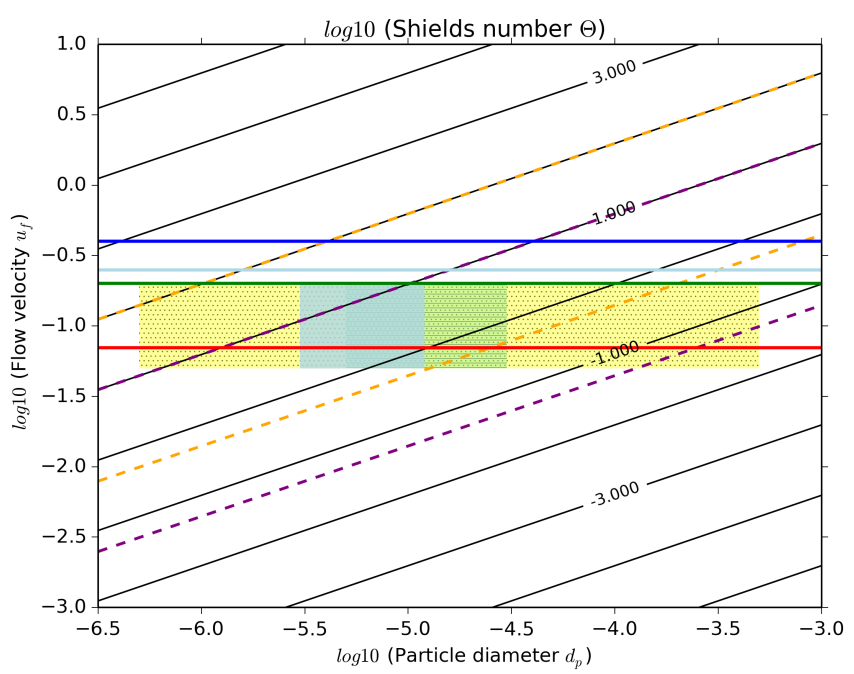

Figure 3. Contour plot of the Shields number as a function of the average flow velocity $\bar{u}_{f}$ and particle diameter $d_{\mathrm{p}}$. The blue, green-striped, and yellow-dotted rectangles show common Dutch, UK, and Australian drinking water conditions, respectively (see Table 2 and the text for explanation). Blue, light-blue, green, and red lines indicate proposed upper limits of self-cleaning velocities of 0.4 (Vreeburg, 2008) and $0.25 \mathrm{~m} \mathrm{~s}^{-1}$ (Blokker et al., 2010), and the default resuspension $\left(0.2 \mathrm{~m} \mathrm{~s}^{-1}\right)$ and deposition $\left(0.07 \mathrm{~m} \mathrm{~s}^{-1}\right)$ velocities for Australian DWDSs (Ryan et al., 2008). As mentioned in the text, the critical Shields numbers for incipient motion, $\theta_{\mathrm{c}}$, are estimated to vary between 0.05 and $10\left(\log _{10}\right.$-values between -1.3 and 1 ). The resuspension threshold is $\theta_{\mathrm{rs}} \approx 10 \theta_{\mathrm{c}}$ (see text) and thus varies between 0.5 and $100\left(\log _{10}\right.$-values of -0.3 and 2$)$.

of of 1 and -1.3 are indicated by the purple lines in Fig. 3. It is emphasized, however, that this latter value needs to be validated empirically to obtain more precise estimates for common conditions in Dutch DWDSs. At an intermediate value of $\theta_{\mathrm{c}}=1$, the associated critical flow velocity is $\bar{u}_{f, c}=0.063 \mathrm{~m} \mathrm{~s}^{-1}$. By comparison, a default, fixed deposition velocity of $0.07 \mathrm{~m} \mathrm{~s}^{-1}$ was derived from experiments on particle transport at conditions common to the Australian DWDSs (Ryan et al, 2008).

Resuspension of particles occurs above a critical wall shear stress for entrainment by the passing fluid, $\theta_{\mathrm{rs}}$. The hydrodynamic forces and torque on a particle sitting on a bed with a destabilizing turbulent flow were developed in a model by Lee and Balachandar (2012). Entrainment occurs when the lift force on a particle exceeds the gravitational force and their model demonstrates that - similar to the threshold for incipient motion - the decrease in $\theta_{\mathrm{rs}}$ is nearly inversely proportional to $R e_{*}$, but values of $\theta_{\mathrm{rs}}$ are approximately an order of magnitude larger than $\theta_{\mathrm{c}}$ for $R e_{*}<1$. The associated thresholds are shown by the orange lines in Fig. 3. At $R e_{*}=1$, the resuspension threshold shear stress is $\theta_{\mathrm{rs}} \approx 1$. By comparison, $\theta_{\mathrm{c}} / \theta_{\mathrm{rs}} \approx 0.1$ is similar to the ratio derived from default fluid velocity thresholds from experiments with drinking water from Australian 
utilities $\left(u_{c}=0.07, u_{\mathrm{rs}}=0.20 \mathrm{~m} \mathrm{~s}^{-1}\right.$; Ryan et al., 2008), i.e., $\left(u_{c} / u_{\mathrm{rs}}\right)^{2}=0.12$. The predictive power of a constant resuspension velocity threshold instead of a variable shear stress threshold may be sufficient for narrow particle diameters and density ranges (such as those of the Netherlands and the UK), but are expected to become inexact for wider parameter ranges.

\subsection{Bed-load transport, flow velocity, and concentration profile}

At moderate shear stresses, a fraction of particles on a bed can mobilize by means of sliding, rolling, and saltating while maintaining contact with the immobile part of the sediment. The sediment bed is not resuspended, but is able to move relative to the fixed substrate at a fraction of the bulk flow velocity. This process is known as bed-load transport and plausibly occurs at wall shear stresses in-between the threshold values for incipient motion, $\theta_{\mathrm{c}}$, and resuspension, $\theta_{\mathrm{rs}}$ (Fig. 1). We apply the commonly used expression for the (non-dimensional) bed-load transport rate, $\phi_{\mathrm{B}}$, known as the Meyer-Peter and Müller equation (Meyer-Peter and Müller, 1948):

$\phi_{\mathrm{B}}=8\left(\theta-\theta_{c}\right)^{3 / 2}$,

Experiments with turbulent water flows over a granular bed in rectangular channels by Florez and De Morales Franklin (2016) validated Meyer-Peter and Müller close to the threshold region. They also demonstrated that far from the threshold region the experimental data better fit a $\left(\theta-\theta_{c}\right)^{5 / 2}$ function, but this was not taken into account in our model, because a comprehensive description is not available. Unsurprisingly, $\phi_{B}$ is a function of the Shields number, and only applies above the threshold shear stress for incipient motion, $\theta_{\mathrm{c}}$. The dimensional volumetric bed-load transport rate per unit width (in the cross-sectional direction of a rectangular channel) is

$q_{B}=\phi_{\mathrm{B}} \sqrt{s g d^{3}}$.

The total volumetric bed-load mass transport rate is Eq. (7) multiplied by the effective channel width, for which we adopt the pipe radius $(D / 2)$ :

$Q_{\mathrm{B}}=q_{B} D / 2$.

The bed-load velocity $u_{\mathrm{B}}$ is related to $Q_{\mathrm{B}}$ as follows:

$u_{\mathrm{B}}=\frac{Q_{\mathrm{B}}}{A_{\mathrm{B}}}=\frac{Q_{\mathrm{B}} \rho_{\mathrm{p}}}{m_{\mathrm{B}}}$,

with $A_{\mathrm{B}}$ the surface of the bed in the cross-sectional direction and $m_{\mathrm{B}}$ the mass per unit length of pipe.
To verify whether bed-load transport operates under DWDS conditions, a sensitivity analysis was performed for a range of common flow velocities, $u_{\mathrm{f}}$, and particle diameters, $d_{\mathrm{p}}$, in a $100 \mathrm{~mm}$ distribution pipe. To determine the dominant transport phases in this parameter space, Shields numbers are calculated and compared to the threshold values for incipient motion $\left(\theta_{\mathrm{c}}\right)$ and resuspension $\left(\theta_{\mathrm{rs}}\right)$ for a range of $d_{\mathrm{p}}$ and $u_{\mathrm{f}}$ values as indicated in Table 3 . The modest influence of flow velocity on the Darcy friction factor was taken into account in the calculation. Further assumptions are a kinematic viscosity of $v=1 \times 10^{-6} \mathrm{~m}^{2} \mathrm{~s}^{-1}$, a relative particle density of $s=\left(\rho_{\mathrm{p}}-\rho_{f}\right) / \rho_{\mathrm{f}}=0.1$, and constant Shields numbers of $\theta_{\mathrm{c}}=1$ and $\theta_{\mathrm{rs}}=10$. Although $\theta_{\mathrm{c}}$ and $\theta_{\mathrm{rs}}$ depend on $u_{\mathrm{f}}, d_{\mathrm{p}}$, and other parameters, as was discussed in Sect. 3.2, the assumed constant values provide a reasonable starting point. Table 3 shows the subdomains for the prevalence of sediment stagnation (bold), bed-load transport (italic), and particle resuspension (bold italic). The results demonstrate that bedload transport is a likely transport mechanism for a range of flow velocities and a broad range of particle diameters that are common in distribution pipes. The domain where bedload transport is dominant shifts to higher flow velocities for larger critical Shields numbers (as can be deduced from Table 3) and for larger pipe diameters (not shown). Note that these results rely on critical Shields numbers that are rough extrapolations from the Shields diagram and need to be validated empirically (Sect. 3.3).

The total volumetric bed-load mass transport rate is calculated using Eq. (8). The results are shown in Fig. 4 and demonstrate that bed-load transport rates increase for progressively larger values of the shear stress (Shields number) and particle diameter. To put these values into perspective, we consider the transport rates of a resuspension event. Strongly discolored water of up to 1000 FTU has been associated with mass concentrations of below $500 \mathrm{mg} \mathrm{L}^{-1}$ (Blokker and Schaap, 2011). Assuming a particle density of $1100 \mathrm{~kg} \mathrm{~m}^{-3}$ and a flow velocity of $0.4 \mathrm{~m} \mathrm{~s}^{-1}$ through a $0.1 \mathrm{~m}$ diameter pipe, the mass transport rate is $1.5 \times 10^{-6} \mathrm{~m}^{3} \mathrm{~s}^{-1}$. Unsurprisingly, transport rates are lower for bed-load transport than for the example resuspension event. Nevertheless, the results suggest that bed-load transport has the potential to contribute substantially to sediment mass transfer in DWDSs. Some care must be taken in the interpretation of the results: if bed-load transport is intermitted by periods of stagnation, the (time-averaged) transport rates decrease accordingly. Also, thin layers or discontinuous patches of sediment likely lower the bed-load transport rates, but this is not accounted for in the formulation that is based on experiments of rectangular channels and thick layers of sediment. Because bed-load transport is likely affected by boundary wall effects and the effective channel width may differ from $D / 2$, transport rates and velocities should be verified for cylindrically shaped pipes.

Below the resuspension threshold, settled and suspended particles coexist. The associated vertical concentration pro- 
Table 3. Ratio of actual Shields numbers to threshold Shields numbers for incipient motion $\left(\theta / \theta_{\mathrm{rs}}\right)$ as a function of particle diameter and bulk flow velocity of water. Color coding refers to the prevalent transport domains (see Fig. 1 and the accompanying text) for each parameter combination: stagnation (bold, $\theta<\theta_{\mathrm{c}}$ ), bed-load transport (italic, $\theta_{\mathrm{c}} \leq \theta<\theta_{\mathrm{rs}}$ ), and resuspension (bold italic, $\theta \geq \theta_{\mathrm{rs}}$ ).

\begin{tabular}{|c|c|c|c|c|c|c|c|c|c|}
\hline & & \multicolumn{8}{|c|}{ Particle diameter (m) } \\
\hline \multirow{10}{*}{ 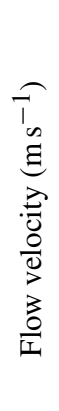 } & & $5.6 \times 10^{-7}$ & $1.0 \times 10^{-6}$ & $1.8 \times 10^{-6}$ & $3.2 \times 10^{-6}$ & $5.6 \times 10^{-6}$ & $1.0 \times 10^{-5}$ & $1.8 \times 10^{-5}$ & $3.2 \times 10^{-5}$ \\
\hline & 0.32 & $2.2 \times 10^{2}$ & $1.8 \times 10^{2}$ & $1.4 \times 10^{2}$ & $1.1 \times 10^{2}$ & $9.2 \times 10^{1}$ & $7.4 \times 10^{1}$ & $6.0 \times 10^{1}$ & $4.8 \times 10^{1}$ \\
\hline & 0.18 & $5.9 \times 10^{1}$ & $4.7 \times 10^{1}$ & $3.8 \times 10^{1}$ & $3.1 \times 10^{1}$ & $2.5 \times 10^{1}$ & $2.0 \times 10^{1}$ & $1.6 \times 10^{1}$ & $1.3 \times 10^{1}$ \\
\hline & 0.1 & $1.6 \times 10^{1}$ & $1.3 \times 10^{1}$ & $1.0 \times 10^{1}$ & $8.3 \times 10^{0}$ & $6.7 \times 10^{0}$ & $5.4 \times 10^{0}$ & $4.3 \times 10^{0}$ & $3.5 \times 10^{0}$ \\
\hline & 0.056 & $4.3 \times 10^{0}$ & $3.5 \times 10^{0}$ & $2.8 \times 10^{0}$ & $2.3 \times 10^{0}$ & $1.8 \times 10^{0}$ & $1.5 \times 10^{0}$ & $1.2 \times 10^{0}$ & $9.5 \times 10^{-1}$ \\
\hline & 0.032 & $1.2 \times 10^{0}$ & $9.5 \times 10^{-1}$ & $7.7 \times 10^{-1}$ & $6.2 \times 10^{-1}$ & $5.0 \times 10^{-1}$ & $4.0 \times 10^{-1}$ & $3.2 \times 10^{-1}$ & $2.6 \times 10^{-1}$ \\
\hline & 0.018 & $3.3 \times 10^{-1}$ & $2.6 \times 10^{-1}$ & $\mathbf{2 . 1} \times 10^{-1}$ & $1.7 \times 10^{-1}$ & $1.4 \times 10^{-1}$ & $1.1 \times 10^{-1}$ & $8.9 \times 10^{-2}$ & $7.2 \times 10^{-2}$ \\
\hline & 0.01 & $9.1 \times 10^{-2}$ & $\mathbf{7 . 3} \times 10^{-2}$ & $5.9 \times 10^{-2}$ & $4.7 \times 10^{-2}$ & $3.8 \times 10^{-2}$ & $3.1 \times 10^{-2}$ & $\mathbf{2 . 5} \times 10^{-2}$ & $2.0 \times 10^{-2}$ \\
\hline & 0.0056 & $2.5 \times 10^{-2}$ & $\mathbf{2 . 0} \times \mathbf{1 0}^{-2}$ & $1.6 \times 10^{-2}$ & $1.3 \times 10^{-2}$ & $1.1 \times 10^{-2}$ & $8.5 \times 10^{-3}$ & $6.9 \times 10^{-3}$ & $5.5 \times 10^{-3}$ \\
\hline & 0.0032 & $\mathbf{7 . 0} \times 10^{-3}$ & $5.7 \times 10^{-3}$ & $4.6 \times 10^{-3}$ & $3.7 \times 10^{-3}$ & $3.0 \times 10^{-3}$ & $2.4 \times 10^{-3}$ & $1.9 \times 10^{-3}$ & $1.5 \times 10^{-3}$ \\
\hline
\end{tabular}

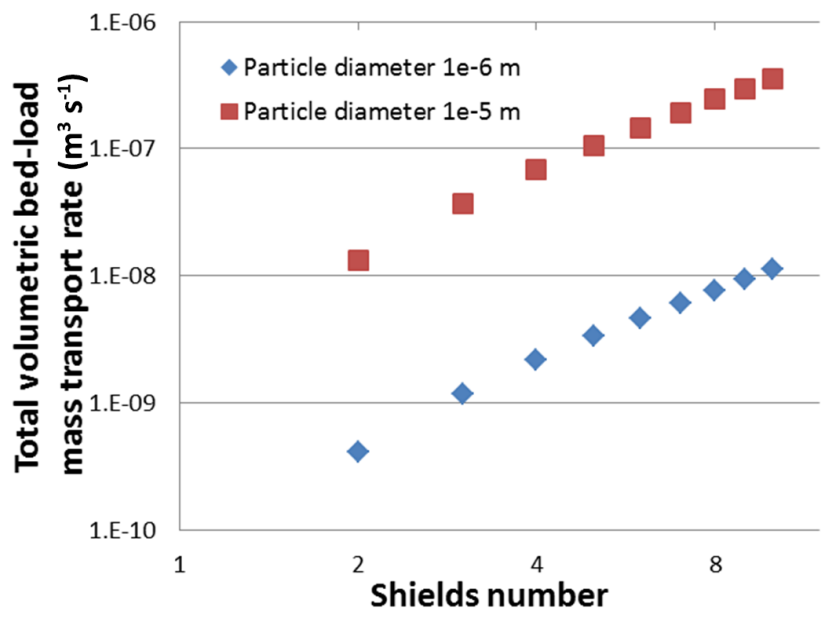

Figure 4. Bed-load transport rate per unit width (in the crosssectional direction of a channel) as a function of the Shields number and for particle diameters as indicated. See the text for parameter values assumed in this calculation.

file and ratio of suspended to bed-load particle mass can be compared to predictions using the non-dimensional Rouse number $P$. The Rouse number is the ratio between settling and upward velocity of suspended particles and is used in fluid dynamics to define the transport mode and vertical concentration profile of suspended sediments (Rouse, 1937):

$P=\frac{u_{\mathrm{s}}}{\kappa u_{*}}$,

with $\kappa$ the von Kármán constant $(\kappa \approx 0.4)$. Experimentally derived $P$-values delimit the transport mode: stagnant sediment $(P>7.5)$, bed-load transport $(2.5<P<7.5), 50 \%$ suspended load $(1.2<P<2.5)$, and $100 \%$ suspended load $(P<1.2)$. We do not consider the condition for wash load $(P<0.8)$, because it does not apply to closed pipes. The
Rouse number also defines the concentration profile, derived from the diffusion equation (Rouse, 1937):

$\frac{c(z)}{c(a)}=\left[\left(\frac{h-z}{x}\right)\left(\frac{a}{h-a}\right)\right]^{P}$,

with $c(z)$ the concentration at height $z$ above the mean bed, $c(a)$ the reference concentration at a reference height $z=a$, and $h$ the water depth. The reference height $a$ is generally taken as $0.05 h$. Equation (11) implies steeper concentration values for larger $P$-values. This is relevant because lance measurements can probe the concentration at different depths and thus have the potential to verify concentration profiles. It should be verified, however, experimentally or theoretically to what extent concentration profiles in closed cylindrically shaped pipes follow Rouse's concentration profile derived for open channels.

\section{Outlook and concluding remarks}

The theoretical assessment presented in this paper serves as a basis for the development of a numerical tool aimed at improving understanding of particle transport and discoloration risk in DWDSs. This requires coupling of the particle transport model to a hydraulic distribution network model to quantify mass accumulation in the sediment and fluid phase. Coupling to a hydraulic model is important because the transport processes in a DWDS are directly influenced by velocity patterns that are, in turn, affected by various factors such as daily, weekly, and seasonal demand fluctuations, network structure (e.g., branched vs. looped layouts), and supply regime. Ultimately, a numerical tool can help to set up more efficient cleaning and measurement programs and assist operators and managers in pipe remediation strategies and the optimization of network designs used in the (re)construction of DWDSs. The presented approach allows for transfer of particulate material between pipe sediments 
across the network and determination of sediment build-up in regions where no measurements are available.

Our analysis shows that for common DWDS conditions, a large range of settling velocities $u_{\mathrm{s}}$, and shear stress thresholds for incipient motion, $\theta_{\mathrm{c}}$, and resuspension, $\theta_{\mathrm{rs}}$, can be expected, because of the strong sensitivity to the average bulk flow velocity $\bar{u}_{f}$, particle diameter, $d_{\mathrm{p}}$, and density, $\rho_{\mathrm{p}}$. The theoretical model predicts $u_{\mathrm{s}}, \theta_{\mathrm{c}}$, and $\theta_{\mathrm{rs}}$ values that are compatible with previously determined values of $\theta_{\mathrm{c}}$ and $\theta_{\mathrm{rs}}$ (or their velocity counterparts) and $u_{\mathrm{s}}$ that were derived from field measurements and modeling for Dutch DWDSs (Vreeburg, 2007; Blokker et al., 2010) or lab experiments with drinking water from Australian utilities (Ryan et al., 2008). However, a model that takes into account these dependencies is expected to result in a more comprehensive and generically applicable model to determine particle accumulation. Further work is required, however, to assess how the influence of turbulent diffusion, dispersion, and transport characteristics based on the Rouse number can be used to improve the particle transport model. Although we primarily focus on hydraulic influences on particles, complex interactions with microbiological and/or chemical processes, as well as the influence of vertical inclinations of pipes, valves, junctions, etc., may hamper successful model predictions. A comparison with field measurements and previously developed models is planned to evaluate and calibrate the model results.

Laboratory and field experiments under controlled conditions are recommended to better understand the complex interplay between particle transport and particle properties, and hydraulic and pipe conditions. Validation of $u_{\mathrm{s}}, \theta_{\mathrm{c}}, \theta_{\mathrm{rs}}{ }^{-}$ values, bed-load transport rates and velocities, and vertical concentration profiles from experiments with pipes of circular cross section is particularly important, because the theoretical relationships considered in this paper are largely derived from experiments using rectangular channels with different boundary wall effects than drinking water pipes.

Use of field measurements in future model development is recommended. For example, vertical turbidity gradients are indicative of bed-load transport as a viable mechanism in DWDSs and can be measured with lancet measurements at multiple pipe heights in the same pipe segment. Another example is that turbidity and flow measurements can provide independent estimates for settling velocity and threshold conditions for incipient motions and resuspension. Extensive field measurements from cleaning actions of several Dutch water companies provide useful data for comparison and validation of the future model results.

Data availability. The results of this paper do not rely on underlying material. The figures and tables have been derived from the equations and explanations in the text. There are no publicly accessible data. As such, we would like to refrain from sharing assets or providing public data access.
Competing interests. The authors declare that they have no conflict of interest.

Special issue statement. This article is part of the special issue "Computing and Control for the Water Industry, CCWI 2016". It is a result of the 14th International CCWI Conference, Amsterdam, the Netherlands, 7-9 November 2016.

Acknowledgements. The study presented in the paper was part of a research project in cooperation with water companies PWN and Evides. The authors thank Peter Schaap (PWN), Henk de Kater (Evides), and Stefan van de Wetering (Brabant Water) for discussions that helped to improve this paper.

Edited by: Edo Abraham

Reviewed by: two anonymous referees

\section{References}

Arolla, S. K. and Desjardins, O.: Transport modeling of sediment particles in a turbulent pipe flow using Euler-Lagrange large eddy simulation, Int. J. Multiphase Flow, 75, 1-11, 2015.

Blokker, E. J. M. and Schaap, P. G.: Sedimentverwijdering bij verschillende snelheden, Report BTO 2006.070, Kiwa Water Research, Nieuwegein, 2007.

Blokker, E. J. M. and Schaap, P. G.: Het modelleren van deeltjes in het leidingnet, Report BTO 2011.047, KWR Watercycle Research Institute, 2011.

Blokker, E. J. M. and Schaap, P. G.: Particle accumulation rate of drinking water distribution systems determined by incoming turbidity, Procedia Engineering 13th Computing and Control for the Water Industry Conference CCWI 2015, Leicester, UK, 2015a.

Blokker, E. J. M. and Schaap, P.: Temperature influences discolouration risk, Procedia Engineering 13th Computing and Control for the Water Industry Conference, CCWI 2015, Leicester, UK, 119, 280-289, 2015b.

Boxall, J. B., Saul, A. J., Gunstead, J. D., and Dewis, N.: A novel approach to modelling sediment movement in distribution mains based on particle characteristics, Water Software Systems, Ch. 1: Theory and Applications, edited by: Ulanicki, B., Coulbeck, B., Rance, J. P., Hertfordshire, UK, 2001.

Blokker, E. J. M., Schaap, P. G., and Vreeburg, J. H. G.: Selfcleaning networks put to the test, Invited papers from the IWA Leading Edge conference on Strategic Asset Management (LESAM), Lisbon, October 2007, edited by: Alegre, H. and Almeida, M. d. C., 2009.

Blokker, E. J. M., Vreeburg, J. H. G., Schaap, P. G., and Van Dijk, J. C.: The self-cleaning velocity in practice, WDSA 2010, Tuscos, AZ, USA, 2010.

Florez, J. E. C. and De Morales Franklin, E.: The formation and migration of sand ripples in closed conduits: Experiments with turbulent water flows, Exp. Therm. Fluid Sci., 71, 95-102, 2016.

Furnass, W. R., Collins, R. P., Husband, P. S., Sharpe, R. L., Mounce, S. R., and Boxall, J. B.: Modelling both the continual erosion and regeneration of dicolouration material in drinking wate distribution systems, Water Sci. Technol., 14, 81-90, 2014. 
Gauthier, V., Barbeau, B., Portal, J.-M., Block, J.-C., and Gatel, D.: Organic matter as loose deposits in a drinking water distribtion system, Water Res., 33, 1014-1026, 1999.

Kris, J. and Hadi, G. A.: CFD investigation of particle deposition and resuspension in a drinking water distribution system, Slovak Journal of Civil Engineering, 1-7, 2007.

Lee, H. and Balachandar, S.: Critical shear stress for incipient motion of a particle on a rough bed, J. Geophys. Res., 117, F01026, https://doi.org/10.1029/2011JF002208, 2012.

Meyer-Peter, E. and Müller, R.: Formulas for bed-load transport, Proc. 2nd Meeting of International Association for Hydraulic Res., 39-64, 1948.

Mounce, S. R., Blokker, E. J. M., Husband, S. P., Furnass, W. R., Schaap, P. G., and Boxall, J. B.: Multivariate data mining for estimating the rate of discolouration material accumulation in drinking water distribution systems, IWA J. Hydroinformatics, 18, 96-114, 2016.

Richardt, S., Korth, A., and Wricke, B.: Model for the calculation of optimised flushing concepts, TECHNEAU: Safe Drinking Water from Source to Tap, edited by: van den Hoven, T. and Kazner, C., IWA Publishing, London, UK, 2009.

Rouse, H.: Nomogram for the settling velocity of spheres, Dev. of Geology and Geography, Exhibit D of the Report of the Commission on Sedimentation, 1936-1937, National Research Council, Washington D.C., 57-64, 1937.

Ryan, G., Mathes, P., Haylock, G., Jayaratne, A., Wu, J., NouiMehidi, N., Grainger, C., and Nguyen, B. V.: Particles in Water Distribution Systems, Report no. 33, The Cooperative Research Centre for Water Quality and Treatment, 2008.

Schaap, P. and Blokker, E. J. M.: Zooming in on network fouling locations, World Environmental and Water Resources Congres, Showcasing the future, Cincinnati, OH, USA, 2013.
Schaap, P. G. and Kivit, C. F. T.: Meten op transportleidingen, Report BTO 2007.003, Kiwa Water Research, Nieuwegein, 2007.

Shields, A.: Anwendung der Ähnlichkeitsmechanik und der Turbulenzforschung auf die Geschiebebewegung, Mitteilungen der Preussischen Versuchsanstalt für Wasserbau und Schiffbau, 26, 1936.

Van Rooij, D. J. M.: Clusteranalyse bruinwatermeldingen Eindhoven, B.Sc. Thesis Dept, Applied Mathematics, Fontys Hogescholen, 2016.

Van Summeren, J., Raterman, B., Vonk, E., Blokker, M., Van Erp, J., and Vries, D.: Influence of temperature, network diagnostics, and demographic factors on discoloration-related customer reports, Procedia Engineering 13th Computing and Control for the Water Industry Conference, CCWI 2015, Leicester, UK, 2015.

Van Thienen, P., Vreeburg, J. H. G., and Blokker, E. J. M.: Radial transport processes as a precursor to particle deposition in drinking water distribution systems, Water Res., 45, 1807-1817, 2011.

Vogelaar, A. and Blokker, E. J. M.: Particle Sediment Modelling, Report BTO 2010.011, KWR Watercyle Research Institute, 2010.

Vreeburg, J. H. G.: Discolouration in drinking water systems: a particular matter, PhD Thesis, Delft University of Technology, 2007

Vreeburg, J. H. G., Schaap, P., and van Dijk, J. C.: Measuring discoloration risk: resuspension potential method, 2nd IWA Leading-Edge on Water and Wastewater treatment technologies, edited by: van Loosdrecht, M. and Clement, J., London: IWA International Water Association, 123-132, 2005. 